
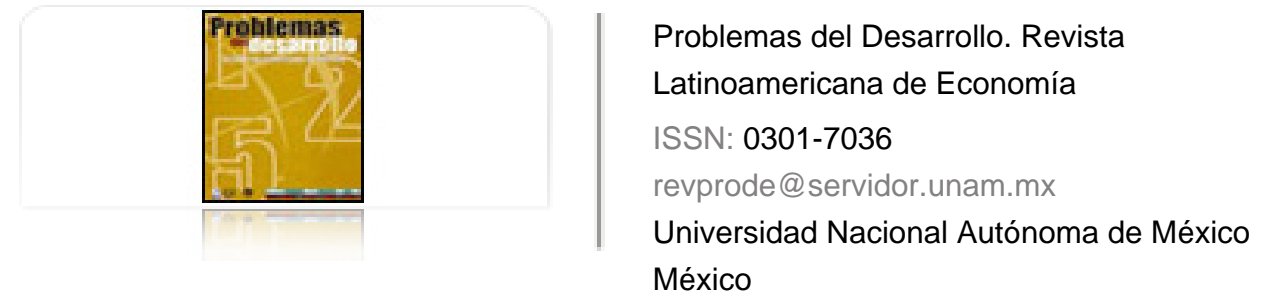

Brugger, Samuel; Ortiz, Edgar

Mercados accionarios y su relación con la economía real en América Latina

Problemas del Desarrollo. Revista Latinoamericana de Economía, vol. 43, núm. 168, enero-marzo, 2012, pp. 63-93

Universidad Nacional Autónoma de México

Distrito Federal, México

Disponible en: http://www.redalyc.org/articulo.oa?id=11822277004

Cómo citar el artículo

- Número completo

- Más información del artículo

Página de la revista en redalyc.org

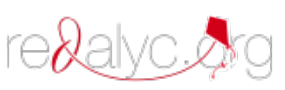

Sistema de Información Científica

Red de Revistas Científicas de América Latina, el Caribe, España y Portugal Proyecto académico sin fines de lucro, desarrollado bajo la iniciativa de acceso abierto 
Revista Problemas del Desarrollo, 168 (43), enero-marzo 2012

\title{
MERCADOS ACCIONARIOS Y SU RELACIÓN CON LA ECONOMÍA REAL EN AMÉRICA LATINA
}

\author{
Samuel Brugger* y Edgar Ortiz**
}

Fecha de recepción: 10 de febrero de 2011. Fecha de aceptación: 24 de septiembre de 2011.

\section{RESUMEN}

Este trabajo examina la relación entre el desempeño de las bolsas latinoamericanas de valores con su economía real aplicando siete modelos econométricos para los casos de Argentina, Brasil, Chile y México. La relación entre los rendimientos bursátiles de estos países y sus respectivos productos internos brutos se examina y compara aplicando para las series de cada país, pruebas de raíz unitaria, análisis de cointegración, análisis del modelo de corrección de error, modelación de causalidad Granger, análisis de vectores autorregresivos, funciones de impulso respuesta, y análisis de descomposición de la varianza. Las series comprenden los índices bursátiles MERVAL de Argentina, BOVEspa de Brasil, IGPA de Chile, e IPC de México compilados de Economática; y las series del producto interno bruto que se obtuvieron de la base de datos CEPALStat. Las series son mensuales y comprenden el periodo 1993-2005.

Palabras clave: Mercados de capital emergentes, desarrollo económico, América Latina.

\section{STOCK MARKETS AND THEIR RELATIONSHIP WITH THE REAL ECONOMY IN LATIN AMERICA}

\begin{abstract}
The paper examines the relationship between the performance of Latin American stock markets with their real economies, applying seven econometric models for the cases of Argentina, Brazil, Chile and Mexico. The relationship between stock market yields in these countries and their respective GDPs is examined and compared, applying to the series for each country unit root tests, cointegration analysis, error correction model analysis, Granger causality modeling, self-regression vector analysis, stimulus and response functions, and variance decomposition analysis. The series comprise the following stock markets: Argentina's MERval, Brazil's BOvESPA, Chile's IGPA, and Mexico's IPC, as compiled by Economática. The GDP series were obtained from the CEPALStat data base. The series are monthly and are for the period 1993-2005.

Keywords: Emerging capital markets, economic development, Latin America.

* Profesor de la Facultad de Economía de la unam e investigador del Instituto Mexicano de Gobernanza Ambiental (IMGM), A.C. Correo electrónico: bruggers@economia.unam.mx

** Profesor Titular "c" de la Division de Estudios de Posgrado de la Facultad de Ciencias Políticas y Sociales de la UNAM, Miembro, sNi III, correo electrónico: edgaro@unam.mx
\end{abstract}


Samuel Brugger y Edgar Ortiz

\section{LES MARCHÉS ACTIONNAIRES ET LEUR RELATION AVEC L'ÉCONOMIE RÉELLE EN AMÉRIQUE LATINE \\ Résumé}

Ce travail examine le rapport entre les performances des bourses latinoaméricaines des valeurs et leur économie réelle, en appliquant sept modèles économétriques pour les cas de l'Argentine, du Brésil, du Chili et du Mexique. Le rapport entre les rendements boursiers de ces pays et leurs respectifs produits intérieurs bruts est examiné et comparé en soumettant les séries de chacun d'eux à des tests de racine unitaire, analyses de co-intégration, analyse du modèle de correction d'erreur, modélisation de causalité Granger, analyse de vecteurs autorégressifs, fonctions de stimulus-réponse, et analyse de décomposition de la variance. Les séries comprennent les indices boursiers MERVAL d'Argentine, BOVESPA du brésil, IGPA du Chili et IPC du Mexique, compilés depuis Economática, et les séries du produit intérieur brut, qui ont été obtenues de la base de données CEPALStat. Les séries sont mensuelles et couvrent la période 1995-2005.

Mots clés : marchés de capital émergents, développement économique, Amérique Latine.

\section{MERCADOS ACIONÁRIOS E SUA RELAÇĀO COM A ECONOMIA REAL NA AMÉRICA LATINA}

\section{Resumo}

Este trabalho examina a relação entre o desempenho das bolsas latino-americanas de valores com sua economia real aplicando sete modelos econométricos para os casos de Argentina, Brasil, Chile e México. A relação entre os rendimentos bursáteis destes países e seus respectivos produtos internos brutos se examina e compara aplicando para as series de cada pais provas de raiz unitária, analise de co-integraçáo, analise do modelo de correçáo do erro, modelagem de causalidade Granger, analise de vetores autoregressivos, funçóes de impulso-resposta, e analise de decomposição da variância. As series compreendem os índices bursáteis MERVAL da Argentina, BOVESPA do Brasil, IGPA do Chile, e IPC do México, compilados de Economática; e as series do produto interno bruto que se obtiveram da base de dados da CEPALStat. As series são mensais e compreendem o período 1993-2005.

Palavras-chave: Mercados de capital emergentes, desenvolvimento econômico, América Latina.

拉丁美洲股票市场及其与实体经济的关系

摘要：

本文以阿根廷、巴西、智利和墨西哥的数据为样本, 采用七个经济计量 模型，检验了拉丁美洲股票市场的表现与实体经济的关系。为检验、比 较上述拉丁美洲国家股票市场表现与GDP的关系, 该研究对每个国家采用 了单位根检验、协整分析、误差修正模型分析、格兰杰因果检验模型、 自回归向量分析、刺激和响应函数、偏差分解分析多个方法。本文采用 股票市场数据序列是由Econom á tica公司编制的阿根廷MERVAL、巴西 BOVESPA、智利IGPA、墨西哥IPC股票市场数据，而GDP数据序列则来自 联合国拉丁美洲经济委员会数据库。本研究使用的数据为月度数据, 时间 跨度为 1993 2005。

关键词：新兴资本市场，经济发展，拉丁美洲 


\section{INTRODUCCIÓN}

Las políticas de liberalización y desregulación financieras instrumentadas en las últimas décadas han dado lugar a una dinámica de la economía mundial concentrada en las actividades de los mercados bursátiles. Múltiples estudios han tratado de determinar el papel de los mercados accionarios en el desarrollo económico. Sin embargo, estas investigaciones han abordado principalmente el caso de los países desarrollados arrojando resultados mixtos. Contribuyendo a este debate el presente estudio analiza la relación a largo plazo entre la actividad bursátil y el crecimiento económico en América Latina (AL) tomando como una muestra representativa los casos de Argentina, Brasil, Chile y México.

\section{MERCADOS BURSÁTILES Y LA ECONOMÍA REAL: TEORÍA Y EVIDENCIA}

Desde los estudios pioneros de Gurley y Shaw (1955, 1960), Goldsmith (1969), Shaw (1973) y McKinnon (1973) importantes estudios sobre finanzas y desarrollo han postulado por décadas una relación positiva entre el sector financiero y el desarrollo económico. Una relación positiva entre los mercados accionarios y el desarrollo económico ha sido recientemente resaltada en la literatura financiera debido al importante crecimiento de estos mercados inducido por la globalización económica y financiera de las últimas décadas. Se propone que el desarrollo de los mercados accionarios promueve el ahorro y que su liquidez coadyuva a sobreponer las limitaciones crediticias prevalecientes en el sector bancario de las economías emergentes. Demirgut-Kunt y Levine (1996) postulan que la liquidez que proviene de los mercados accionarios incrementa la productividad del capital a un nivel global de la economía y facilita la inversión rentable a largo plazo. Levine (1997) y Mishkin (2001) afirman que un sistema financiero bien desarrollado, incluyendo en él a los mercados de capital, alienta la inversión gracias a la mejor identificación y financiamiento de oportunidades lucrativas de negocios, la movilización del ahorro, la eficiente asignación de recursos, la diversificación del riesgo, y su apoyo al intercambio de bienes y servicios. Enisan y Olufisayo (2009) revisando una extensa literatura documentan varios canales a través de los cuales el desarrollo de los mercados financieros contribuye al desarrollo económico. Enisan y Olufisayo identifican cinco mecanismos: 1) mayor eficiencia en la asignación del capital como proporción del ahorro financiero ya que la riqueza total se incrementa; 2) movilización del ahorro al ofrecer más atractivos instrumentos y vehículos de ahorro; 3) provisión de 
instrumentos para negociar, conjuntar y diversificar el riesgo; 4) disminución de los costos de adquisición y procesamiento de la información lo que mejora en la asignación de los recursos, y 5) incremento en la especialización productiva, desarrollo del emprendurismo, y la adopción de nuevas tecnologías.

Sin embargo muchos puntos de vista contradicen los principios de las finanzas tradicionales. Stiglitz (1985) y Capasso (2004) afirman que mercados de capital desarrollados pueden convertirse en ineficientes mecanismos para la adquisición de información para la toma de decisiones de inversión tanto de cartera como real, debido a la naturaleza de la información pública de noticias buenas y malas disponible en estos mercados. En el caso de los mercados accionarios emergentes la falta de información puede conllevar a decisiones ambiguas tanto por parte de los inversionistas, como de los empresarios. Aún más, en ambos tipos de mercados la presencia de información asimétrica inhibe la inversión; las políticas sobre tasas de interés y tipo de cambio se vuelven ineficaces en tanto que las decisiones bancarias y corporativas son subóptimas porque sus tomadores de decisiones no pueden distinguir entre buenos y malos deudores. Bajo estas circunstancias la banca y empresas afrontan políticas crediticias restrictivas como son el racionamiento del crédito y restricciones cuantitativas impuestas por los inversionistas de los mercados de deuda y accionarios (Stiglitz y Weiss, 1981). Igualmente Singh (1997) y Singh y Weiss (1998) apuntan que el desarrollo financiero puede no ser favorable para el desarrollo económico, destacando tres razones: primera, la inherente volatilidad y arbitrariedad de los procesos de fijación de precios en los mercados accionarios emergentes, lo que los convierte en seńales deficientes para la asignación de recursos para las inversiones; segunda, los vínculos entre los mercados accionarios y los mercados de divisas pueden exacerbar la inestabilidad económica y reducir el crecimiento a largo plazo cuando ocurren shocks desfavorables; finalmente, el desarrollo de los mercados de capital puede debilitar las fuertes relaciones tradicionalmente establecidas entre la banca y empresas en los países en vías de desarrollo, conllevando a sensibles limitaciones en la inversión real.

La evidencia sobre los mercados accionarios y el desarrollo es abundante y controvertida. En un estudio pionero, Fama (1990) utiliza la producción futura para explicar, con distintas frecuencias, los rendimientos bursátiles entre 1953 y 1987 en Estados Unidos. Su estudio demuestra que la economía real es la causa de los rendimientos bursátiles, lo que difiere con la mayoría de los demás trabajos, que sugieren una relación inversa. Más directamente, el trabajo de Hassapis y Kalyvitis (2002), identifica relaciones positivas en los cambios de los precios de las acciones y la tasa de crecimiento de los países de la OCDE para el periodo comprendido entre 1950 y 1990 en la mayoría de los países de la OCDE, excepto 
en Italia y Francia. Nasseh y Strauss (2000) también presentan evidencias de una relación de largo plazo entre los precios accionarios y la producción industrial de las mayores economías europeas.

Binswanger (2000, 2004a, 2004b), por su parte, presenta un análisis semejante al de Fama (1990) pero centrándose en la producción industrial y en los rendimientos bursátiles de Estados Unidos durante 1950 y 1990. Investiga varios periodos, pero independientemente de la frecuencia analizada y, mediante la implementación de la prueba de Chow de cambio estructural, el autor demuestra que el rendimiento de las acciones ha dejado de ser un buen indicador líder de la actividad económica de la economía real desde los años ochenta, es decir, desde el incremento de flujos de capital financiero a nivel global. En un estudio reciente, Athanasios y Adamopoulos (2009) empleando un modelo de causalidad de Granger demuestran que en el caso de Francia el desarrollo financiero tiene un impacto positivo en el desarrollo económico, en tanto que las tasas de interés tienen un efecto negativo en el mercado accionario.

Para los países con mercados bursátiles subdesarrollados existen pocos estudios y la evidencia es todavía más contradictoria. Atje y Jovanovic (1993) en un estudio de corte transversal de más de 40 países presentan evidencia sobre la presencia de correlación entre la media de crecimiento económico y la capitalización de los mercados bursátiles. Levine y Zervos (1998) extienden el estudio incluyendo más variables y alargando el periodo de estudio, encontrando que la liquidez del mercado bursátil está muy relacionada con el crecimiento económico, la acumulación de capital y la productividad, pero no con el tamaño del mercado de capital. Mauro (2003) buscó la correlación entre el crecimiento de la producción y los rendimientos bursátiles con rezagos en un panel de economías emergentes y desarrolladas. Su estudio demostró que la proporción de países desarrollados y subdesarrollados en los cuales la correlación es significante es la misma si se utilizan datos anuales, pero no es significante al utilizar datos trimestrales. Caporale, Howells y Soliman (2003 y 2005) emplean un modelo VAR para mostrar causalidad y obtuvieron como resultado que el desarrollo del mercado bursátil mejoró el crecimiento económico de largo plazo en Chile, Corea, Malasia y Filipinas, principalmente por los impactos de la inversión. Choong, Yusop, Law y Sen (2003) demostraron, mediante distribuciones autorregresivas con retrasos (ARDL) y causalidad de Granger, que existe cointegración entre el desarrollo de los mercados bursátiles y el crecimiento económico para Malasia durante 1978-2000. Nyong (1997) usó un índice de desarrollo de mercado de capital para Nigeria y encontró que el desarrollo está negativamente correlacionado con el crecimiento de largo plazo de ese país. 
Samuel Brugger y Edgar Ortiz

En resumen, la teoría tradicional de finanzas y desarrollo postula una relación directa entre el sector financiero y el desarrollo económico. Esta teoría ha sido extendida recientemente para sugerir una relación directa entre el desempeño de los mercados accionarios y el crecimiento económico. En ambos casos se han adelantado posiciones alternativas y la evidencia empírica ha producido resultados que validan las dos posiciones o que demuestran la presencia de un impacto bidireccional entre finanzas y desarrollo. De todos modos, la evidencia empírica manifiesta la necesidad de profundizar la investigación sobre los vínculos entre mercados accionarios y desarrollo a largo plazo, en particular para el caso de las economías emergentes incluyendo en este tema a los países latinoamericanos para los cuales la literatura financiera es reducida.

\section{DATOS Y METODOLOGÍA}

\section{Series de tiempo}

Para examinar la relación de largo plazo entre los mercados accionarios y la economía real para los casos de Argentina, Brasil, Chile y México, el presente estudio utiliza series de tiempo mensuales de sus pIB y de los índices de sus bolsas de valores para el periodo 1993.1 a 2005.12. El conjunto de series temporales financieras se obtuvo de la base de datos Economática y el conjunto de series temporales económicas, esto es el piв de cada país se obtuvo de la base de datos CEPALStat. Las series financieras comprenden los índices bursátiles de Argentina (MERVAL), Brasil (Bovespa), Chile (IGPA), y México (IPC). Tanto las series financieras como las correspondientes a la economía real se homogenizaron a una base de $100=1993$. Las series comprenden un total de 196 observaciones.

\section{Modelación econométrica}

El marco metodológico a aplicarse consiste de seis modelos econométricos a fin de desarrollar una visión comprensiva sobre la relación entre mercados bursátiles y desarrollo económico en AL; estas pruebas son: $a$ ) análisis de raíces unitarias; b) análisis de cointegración, c) prueba de mecanismo de corrección de error, $d$ ) modelo de causalidad de Granger, $e$ ) funciones de impulso respuestas, y $f$ ) análisis de vectores autorregresivos.

Previamente a cualquier prueba econométrica es preciso determinar si las series son estacionarias a fin de no concluir erróneamente proponiendo relaciones 
espurias entre las variables bajo análisis, problema que se suscita en series financieras con tendencias crecientes alrededor de su media y varianza en el tiempo. Así, a fin de determinar si existe una relación de equilibrio de largo plazo entre los mercados accionarios y la economía real es preciso descartar la presencia en las series de raíces unitarias y realizar pruebas de cointegración. La prueba de raíz unitaria de Dickey-Fuller Aumentada para una serie temporal consiste de una regresión de las primeras diferencias de la serie como variable dependiente y de $\mathrm{k}$ rezagos de la serie temporal como variables independientes:

$\Delta y=\alpha+\beta t+\delta y_{t-1}+\sum_{j=1}^{k} \delta \Delta y_{t-j}+\varepsilon_{t}$

donde $\Delta y_{t}=y_{t}-y_{t-1}$ y $\Delta y_{t}=y_{t}-y_{t-1} ; \beta$ es el coeficiente de la tendencia en el tiempo y $\mathrm{k}$ el orden del proceso autorregresivo. Los coeficientes $\alpha$ y $\beta$ deben ser igual a cero indicando un modelo de caminata aleatoria con desvío. Las hipótesis nula y alternativa son $H_{0}: \delta=0 ; H_{1}: \delta<0$. La aceptación de la hipótesis nula indica no estacionaridad. Finalmente, el orden de los rezagos permite realizar esta prueba por un proceso regresivo de orden $n$. El número de rezagos debe por tanto ser determinado. Los resultados se obtienen comparando el valor obtenido con los valores críticos de los niveles de confianza deseados. El número obtenido es negativo mientras más grande sea más fuerte es el rechazo de la existencia de raíz unitaria en la serie logarítmica analizada; alternativamente se usa la probabilidad p de un lado de McKinnon; mientras más chica es dicha probabilidad más fuerte es el rechazo a la hipótesis nula.

Una vez que se ha establecido la presencia de estacionaridad de las series, puede procederse a comprobar si los pares de series de cada país tienen diferentes raíces unitarias, lo que significaría ausencia de cointegración, o si por el contrario las series comparten la misma raíz unitaria, comprobándose así la presencia de cointegración y por tanto de una relación de equilibrio de largo plazo con el cual el sistema converge con el paso del tiempo. Si las variables cointegradas sufren una perturbación el desequilibrio es sólo de corto plazo ya que las fuerzas del mercado juegan un papel importante para impedir divergencias persistentes.

La idea central de la cointegración indica que si para dos variables existe un vector $[1-\beta]^{\mathrm{T}}$ que hace posible establecer una relación lineal entre las dos variables, $X_{t}-\alpha-\beta Y_{t}=Z_{t}$ sea del orden $\mathrm{I}(0)$, entonces las dos variables están cointegradas en el orden $(1,1)$. La relación lineal se denomina regresión de cointegración donde " $\beta$ " es un parámetro de cointegración y $\mathrm{Z}$ corresponde a un error de equilibrio y mide la magnitud de la desviación de corto plazo respecto al equilibrio de largo plazo. La prueba de cointegración por tanto consiste de un análisis de los residuos de la ecuación de integración para que satisfaga el 
requisito de que el residuo de la regresión sea $\mathrm{I}(0)$. El número de rezagos óptimo debe ser determinado previamente. Una prueba importante de muy común aplicación para determinar la presencia de cointegración en series financieras es la prueba de Johansen $(1991,1995)$. Concretamente, considérese un var del orden $\mathrm{p}$ :

$y_{t}=A_{1} y_{t-1}+A_{p} y_{t-p}+B x_{t}+\varepsilon_{t}$

donde $\mathrm{y}_{\mathrm{t}}$ es el vector $\mathrm{k}$ de las variables mensuales $\mathrm{I}(1)$ no estacionarias; $\mathrm{x}_{\mathrm{t}}$ es un vector de variables determinísticas; y $\varepsilon_{\mathrm{t}}$ es un vector de innovaciones. Este VAR puede re-expresarse como se indica en las siguientes ecuaciones:

$$
\begin{aligned}
& \Delta y_{t}=\Pi y_{t-1}+\sum_{p=1}^{p-1} \Gamma_{t} \Delta_{t-1}+B x_{t}+\varepsilon_{t} \\
& \Pi=\sum A_{t}-I: \Gamma_{t}=-\sum_{j=i+1}^{p} A_{j}
\end{aligned}
$$

De acuerdo a la representación del teorema de Granger (1969), si la matriz de coeficientes $\Pi$ tiene un rango reducido de $\mathrm{r}<\mathrm{k}$, entonces existe $\mathrm{k}(\mathrm{r})$ matrices $\alpha$ y $\beta$, cada una con un rango r de tal manera que $\Pi=\alpha \beta^{\prime}$ y $\beta^{\prime} y_{t} \mathrm{I}(0)$. El número de relaciones cointegrantes está dado por r, i.e. el rango de cointegración. Finalmente, cada columna de $\beta$ es el vector cointegrante; si $r=0$, no existen vectores cointegrantes. Los elementos $\alpha$ son los parámetros de ajuste en el vector de corrección de errores. La técnica de Johansen estima la matriz $\Pi$ de una manera no restrictiva, y entonces prueba de rechazar la restricción implicada por el rango reducido de $\Pi$.

Dos pruebas están asociadas con el modelo de Johansen: La prueba de la tra$z a$ y la prueba del máximo eigenvalue. La prueba de la traza es una prueba conjunta; la hipótesis nula indica que el número de vectores cointegrantes es menor o igual a r, en tanto que la hipótesis alternativa indica que hay más vectores cointegrantes que r. La prueba del máximo eigenvalue realiza pruebas separadas para cada valor de eigen. La hipótesis nula señala que existen $r$ vectores cointegrantes, en tanto que la hipótesis alternativa indica que hay $\mathrm{r}+1$ vectores cointegrantes.

Aunque dos variables, como son los rendimientos del mercado accionario y el pIB mantengan una relación de equilibrio de largo plazo, es posible encontrar desviaciones de corto plazo. Para determinar la magnitud de tal desequilibrio y su ajuste de un periodo a otro, es necesario aplicar el mecanismo de corrección de errores (ECM) de Sargan (1964; 2000) y Engle y Granger (1987). Al respecto, es importante resaltar que un error rezagado estimado significativo indica 
la presencia de importantes canales de influencia entre las variables analizadas porque en tal situación las series se ajustan rápidamente de cualquier desvío del equilibrio de largo plazo que comparten las variables. En general, este modelo sólo incluye un rezago de la diferencia de las variables independientes. El presente estudio incluye rezagos adicionales pero probando su significancia con pruebas de correlación serial, correlograma de heterocedasticidad, Q-tests, y pruebas de residuos. Los siguientes modelos genéricos de ЕСм se aplican tanto para el PIB, como para los índices bursátiles de cada país para demostrar los canales de influencia entre estas dos variables.

$\Delta P I B_{t}=\beta_{1}+\beta_{2} \hat{e}_{t-1}+\sum_{j=1}^{12} \beta_{3 \mathrm{j}} \Delta P I B_{t-j}+\sum_{j=1}^{12} \beta_{4 \mathrm{j}} \Delta I B_{t-j}+\xi_{t j}$

$\Delta I B_{t}=\beta_{1}+\beta_{2} \hat{e}_{t-1}+\sum_{i=1}^{12} \beta_{3 \mathrm{i}} \Delta P I B_{t-i}+\sum_{j=1}^{12} \beta_{4 \mathrm{i}} \Delta I B_{t-i}+\xi_{t i}$,

donde,

$\triangle P I B=$ Cambio contemporáneo en el PIB (de cada país)

$\triangle I B_{t}=$ Cambio contemporáneo en el índice bursátil de cada país

$\xi=$ Representa el tamaño del residuo derivado de la combinación lineal de las dos variables cointegradas, también conocida como desviación del equilibrio a largo plazo o término de corrección de error.

La causalidad Granger explica si x causa y, esto es, cuánto del valor corriente de y puede explicarse por valores pasado de $y$ para entonces ver si ańadiendo valores rezagados de $x$ se puede mejorar la explicación sobre el comportamiento de $y$, en este caso se dice que $y$ es Granger causado por $x$ si $x$ coadyuva en la predicción de $y$; de manera equivalente, esta afirmación es válida si los coeficientes de las variables rezagadas $x$ 's son estadísticamente significativos. Frecuentemente se encuentra una relación bilateral de causalidad: $x$ Granger causa $y$, en tanto que también $y$ Granger causa $x$. El modelo puede representarse como sigue:

$$
\begin{aligned}
& y_{t}=\alpha_{1} y_{t-1}+\mathbf{L}+\alpha_{l} y_{t-l}+\beta_{1} x_{t-1}+\mathbf{L}+\beta_{l} x_{t-l}+\varepsilon_{t} \\
& x_{t}=\alpha_{1} x_{t-1}+\mathbf{L}+\alpha_{l} x_{t-l}+\beta_{1} y_{t-1}+\mathbf{L}+\beta_{l} y_{t-l}+\varepsilon_{t}
\end{aligned}
$$

Aquí $l$ indica el número de rezagos.

La metodología de vectores autorregresivos (VAR) es un importante completente del análisis econométrico contemporáneo. Estos modelos son una 
generalización del modelo autorregresivo AR; en general una variable se explica en función de sus valores pasados y su amplio uso se debe a la simplificación de muchos supuestos y restricciones que pedían los modelos de índole estructural. La simplificación más relevante es que deja de existir la asignación a priori entre variables endógenas y exógenas, y recupera de esta manera la dinámica original de las series de tiempo. No es necesario establecer con anticipación la condición de exogeneidad-endogeneidad, pues define todas las variables como endógenas. Esto es relevante en estudios como en el presente, donde existe interdependencia en todo momento entre sus series. El modelo var generalizado sin restricciones de Sims (1980), aplicado en el presente estudio, consiste en regresiones de cada una de las variables no rezagada respecto a todas las demás con varios rezagos a partir de:

$$
\begin{array}{r}
Z_{t}=\sum_{i=1}^{k} A_{i} Z_{(-i)}+\varepsilon_{i} \\
\text { donde } Z_{t}=\left(\begin{array}{l}
y_{t} \\
x_{t}
\end{array}\right)
\end{array}
$$

los rezagos; $\mathrm{y}_{t}, \mathrm{y} x_{t}$ indican endogeneidad en las especificaciones. También se asume que $\varepsilon_{t}$ es un vector columna de errores aleatorios o innovaciones que se considera están contemporáneamente correlacionados pero no autocorrelacionados, por lo que no tienen una matriz de covarianzas diagonal (Charemza y Blangiewicz, 2001). Como los modelos var sólo tienen variables rezagadas del lado derecho y éstas no van correlacionadas con el término de error, se estima cada ecuación mediante mínimos cuadrados ordinarios. Para explicar los cambios en el piB en función de sí mismo y de los rendimientos bursátiles el presente estudio aplica la siguiente regresión para cada uno de los países;

$$
\begin{gathered}
\begin{array}{c}
\Delta P I B=\alpha_{0}+\alpha_{1} \Delta P I B_{t-1}+\alpha_{2} \Delta P I B_{t-2}+\ldots .+\alpha_{11} \Delta P I B_{t-l l}+\beta_{1} \Delta I B_{t-1} \\
+\beta_{2} \Delta . I B_{t-2}+\ldots .+\beta_{11} \Delta I B_{t .11}+\varepsilon_{t}
\end{array} \\
\begin{array}{c}
\Delta I B=\alpha_{0}+\alpha_{1} \Delta I B_{t-1} \\
+\alpha_{2} \Delta I B_{t-2}+\ldots . .+\alpha_{11} \Delta I B_{t-l l}+\beta_{1} \Delta P I B_{t-1} \\
+\beta_{2} \Delta . P I B_{t-2}+\ldots .+\beta_{11} \Delta P I B_{t . l 1}+\varepsilon_{t}
\end{array}
\end{gathered}
$$

Un factor determinante es el número de rezagos que debe aplicarse por lo que es necesario evaluar uno a uno cada rezago hasta encontrar el número óptimo. Para ello se vuelve indispensable la contrastación de los criterios de información estadística con los resultados que genera cada especificación.

Las ecuaciones de impulso respuesta se obtienen estimando un modelo (VAR). El modelo examina las interrelaciones dinámicas y relaciones de causalidad entre 
la actividad bursátil y el PIB identificando los shocks en un sistema cointegrado. La estimación VAR es una generalización multivariada de la causalidad de Granger. El comportamiento dinámico del modelo var puede ser caracterizado graficando las funciones de impulso-respuesta lo cual determina cómo responde cada variable endógena en el tiempo en esa variable y en otras variables endógenas. Sin embargo, el impulso-respuesta puede ser calculado sólo si el modelo es estable en un equilibrio a largo plazo; ahí, shocks en un periodo de la variable endógena afectan las otras variables endógenas. Graficando la función de impulso-respuesta se sintetiza la estructura dinámica del sistema. Para llevar a cabo los análisis empíricos acerca de los cambios en los mercados emergentes de AL y los PIB de sus respectivos países este trabajo aplica un modelo var bivariado para cada caso:

$$
\begin{aligned}
& \Delta P I B_{t}=\beta_{1}+\beta_{2} \Delta P I B_{t-1}+\beta_{3} \Delta I B_{t-1}+\xi_{t 1} \\
& \Delta I B_{t}=\alpha_{1}+\alpha_{2} \Delta P I B_{t-1}+\alpha_{3} \Delta I B_{t-1}+\xi_{t 2}
\end{aligned}
$$

El análisis impulso-respuesta es un instrumento útil para evaluar la congruencia y la sensibilidad dinámica de las variables especificadas en el modelo. Sin embargo, hay que cuidar de no tener covarianzas distintas a 0 , es decir, errores correlacionados, ya que en ese caso sería imposible precisar la respuesta de las variables ante impulsos de variables específicas (Pindyck y Rubinfeld, 2001). El análisis indica la respuesta dinámica de la variable dependiente en el var ante choques en los términos de error o innovaciones de todas las variables endógenas, excluyendo los efectos de las variables que expresamente asignamos como exógenas. Algo relevante es que la respuesta al impulso sólo puede ser calculada si el var se encuentra en equilibrio de largo plazo. Por otro lado, se debe considerar la longitud del choque, ya que si éste se considera muy corto no se pueden observar con precisión la evolución de los choques ni la estabilidad dinámica del var. De esta manera, si el var es estable, una perturbación hará que el sistema salga de su trayectoria de equilibrio, aunque después de algunos periodos se restablezca. Una característica de este análisis es que, si se aplica la metodología original de Cholesky, el orden en la asignación de las variables es muy importante, ya que influye directamente sobre los resultados. Es decir, el modelo resuelve siguiendo la línea de causalidad que se asignó en la especificación. Sin embargo, en el presente caso se puede dejar de lado, ya que sólo se hará un análisis basado en dos variables. 
Samuel Brugger y Edgar Ortiz

\section{RESULTADOS EMPÍRICOS}

\section{Desempeño bursátil y crecimiento en los mercados emergentes de AL}

Previo al rigor de los análisis econométricos conviene examinar los patrones de cambio de la economía real y de las bolsas de valores de los países incluidos en el presente estudio. Tanto el pIB como el sector bursátil de Argentina, Brasil, Chile y México experimentaron un crecimiento en general irregular y en términos regionales heterogéneo. La Gráfica 1 resume el desempeño del piв de los cuatro países estudiados. Puede observarse que con excepción de Chile, ninguno de los países tuvo un crecimiento continuo durante el periodo analizado. Resaltan los recurrentes ciclos de auges y caídas de Argentina, Brasil y México, pero con marcadas diferencias en su volatilidad; resaltan también las crisis de la economía mexicana de 1994-1995, la crisis brasileńa de 1998-1999 y el desplome de la economía argentina de 2001-2002.

Un primer acercamiento a la relación entre los mercados accionarios, representados por sus índices bursátiles medidos en dólares y sus respectivos PIB igualmente medidos en dólares se resume en la Gráfica 2. Difícilmente se puede reconocer alguna relación entre estas variables. En ningún caso se puede hablar de relación alguna, excepto del caso chileno, donde se observa una relación muy ligera de la tendencia de crecimiento. Sin embargo, ésta no es una prueba definitiva pues como se detalló en la modelación econométrica esta relación debe ser medida utilizando las primeras diferencias de las series temporales, lo que se reporta en un apartado posterior.

Gráfica 1. PIB mensual de las principales economías latinoamericanas (1993=100)
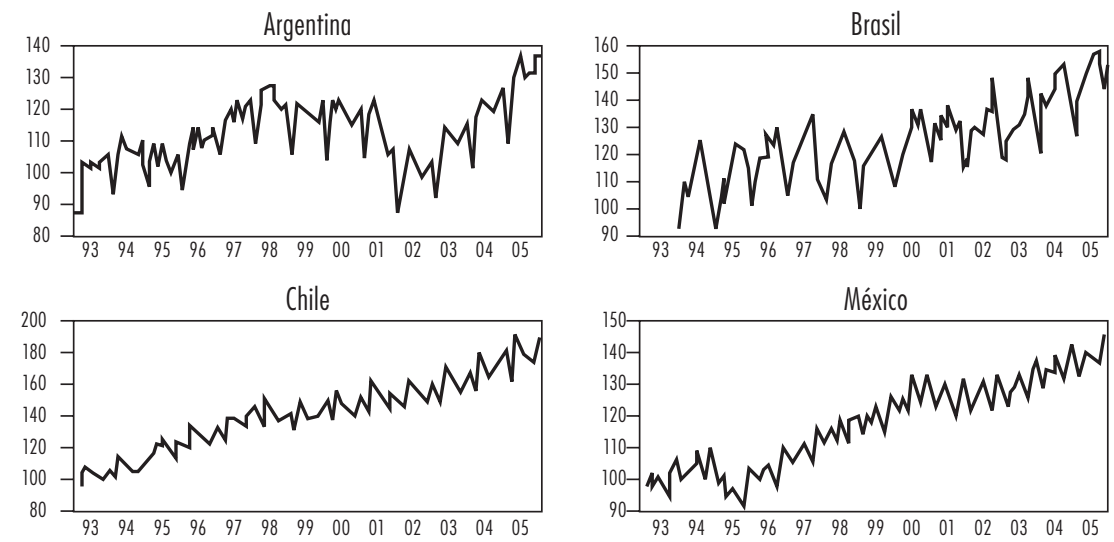

Fuente: Elaboración propia con base en: CEPalStat (Estadísticas de América Latina y El Caribe). 
Gráfica 2. Relación PIB-Índices bursátiles en América Latina
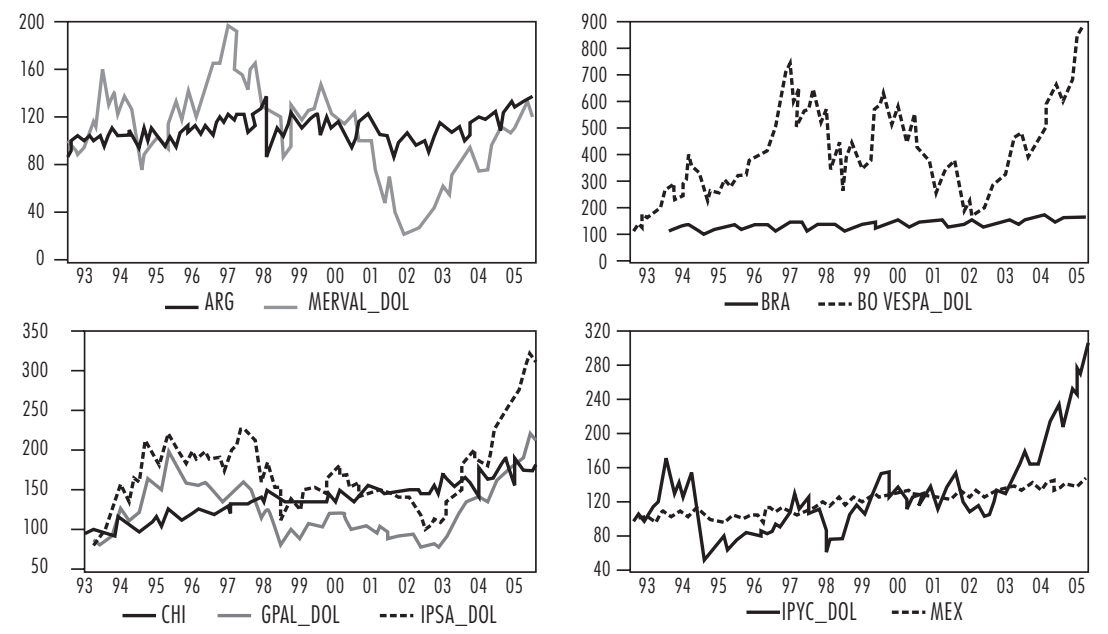

Fuente: Elaboración propia con base en: CEPALStat (Estadísticas de América Latina y El Caribe).

\section{Estadísticas básicas}

Las principales característica estocásticas del crecimiento del pIB y del rendimiento de los índices accionarios de Argentina, Brasil, Chile y México homogenizando sus tendencias en dólares de Estados Unidos a fin de realizar un análisis comparativo y en términos de una visión de los inversionistas y agentes económicos internacionales, pueden resumirse como sigue. ${ }^{1}$

Partiendo de la base común 100=enero 2 de 1993, en el panel a del Cuadro 1 puede apreciarse que la economía chilena experimentó el crecimiento más alto de los cuatro países; su promedio mensual para el periodo bajo análisis (1993-2005) fue de 0.59\%. En escala descendiente siguen después el PIB de Brasil (0.33\%), el pIB de Argentina (0.32\%) y con el menor crecimiento el piB de México (0.31\%). La dispersión en el crecimiento de la producción agregada de las principales economías de AL no siguen pari pasu el desempeño global del crecimiento del pIB. Los dos primeros lugares se invierten; la desviación estándar del crecimiento del pIB de Brasil es la mayor (8.129 puntos),

1 El cuadro sobre las estadísticas básicas están disponibles contactando a los autores. 
seguido por la desviación estándar de Chile (7.697 puntos), las dos últimas posiciones también se invierten; el tercer lugar lo ocupa Argentina cuya desviación estándar del crecimiento del piB es de 4.08 puntos, en tanto que la volatilidad del crecimiento del PIв de México registra una desviación estándar de 6.413 puntos.

El estadístico sobre la asimetría en Cuadro 1, panel a, revela que las series del crecimiento del pIB para los casos de Brasil, Chile y México se caracterizan por un sesgo positivo en tanto que Argentina presenta un sesgo negativo bajo; el sesgo positivo mayor es el de Chile (0.1.286) y el sesgo negativo del crecimiento de Argentina es de sólo - 0.285 puntos. En cuanto a la curtosis destaca el hecho que en los casos de las distribuciones de las series del crecimiento de los PIB de Argentina, Chile son leptocúrticas (su valor es mayor a 3), es decir se trata de distribuciones puntiagudas. La distribución del crecimiento del PIB de México es claramente mesocúrtica, esto es, un tanto aplanada en relación a la curva normal; el caso de Brasil se encuentra en la frontera inferior de una distribución leptocúrtica. Finalmente, midiendo la normalidad de las series, de acuerdo con la Prueba Jarque-Bera del Cuadro 1, panel a, se observa que en los casos del crecimiento de los piB de Argentina y Chile sus estadísticos son mayores al valor crítico de 5.9 al nivel de confianza de 5.0\%, indicando la ausencia de normalidad; el crecimiento de Chile es el que más se aleja de la normalidad. La Prueba de Jarque-Bera sugiere normalidad en los casos de Brasil y México.

Examinando los rendimientos, el mercado accionario de Sao Paulo fue el más rentable debido a su crecimiento inflacionario, registrando un rendimiento mensual de $5.03 \%$. En escala descendente se encuentran los rendimientos de México $(1.37 \%)$, Chile $(0.75 \%)$, y registrando el menor crecimiento el merval de Argentina (0.11\%). El comportamiento de la volatilidad es un poco diferente. En primer lugar se encuentra el mercado brasileńo con una desviación estándar de 51.48 puntos, seguido por el MERval de Buenos Aires (11.92 puntos), luego el IPC de México (11.52 puntos), y finalmente como menos disperso el mercado accionario chileno (7.57). Estas medidas indican la presencia de una alta volatilidad en los mercados de capitales de AL con una gran diferencia en la relación rendimiento-riesgo entre estas bolsas regionales. Efectivamente, en función de la desviación estándar el rendimiento por puntos de riesgo asumido medido por el coeficiente rendimiento/desviación estándar es mejor en el caso de la Bolsa Mexicana de Valores (0.119\%), seguido de los casos de Chile (0.0989\%) y Brasil $(0.0977 \%)$ y en el último lugar la bolsa argentina $(0.00977 \%)$.

Respecto a la asimetría destaca el hecho de que tres de los cuatro mercados analizados tienen un sesgo negativo (Argentina, Brasil y México) y sólo 
el mercado accionario de Santiago de Chile se caracteriza por un sesgo positivo, sugiriendo de todos modos y en todos los casos distribuciones alejadas de la distribución normal. Este hecho se confirma con el estadístico sobre la curtosis que para todos los mercados bajo análisis es mayor a 3 indicando la existencia de distribuciones puntiagudas. No obstante, en el caso de Chile, cuya curtosis es de 3.5, la Prueba de Jarque-Bera no rechaza la ausencia de normalidad. En todos los demás mercados accionarios este estadístico es mayor al valor crítico de 5.9 confirmando la ausencia de normalidad. En el caso de Chile la aparente contradicción puede explicarse por la presencia del sesgo positivo no tan alejado de cero e igualmente la curtosis no tan elevada identificada anteriormente.

\section{Actividad bursátil y desarrollo en América Latina}

\section{Análisis de raíces unitarias}

Para analizar las relaciones entre dos o más variables en necesario precisar si los procesos estocásticos que generaron las series de tiempo son invariables en el tiempo, esto es, si dichos procesos son estacionarios (ec. 1). Se requiere que las series carezcan de raíz unitarias y que sean por tanto del orden $\mathrm{I}(0)$; las series con un orden $\mathrm{I}(\mathrm{d})>0$ tienen al menos una raíz unitaria. La prueba más común aplicada en la economía financiera para identificar la presencia o ausencia de raíces unitarias es la de Dickey-Fuller Aumentada (ADF), complementada con el valor crítico de los valores de probabilidad de un lado (one sided p-values) de MacKinnon (1996). El Cuadro 1 muestra los resultados de esta prueba para el PIB de Argentina, Brasil, Chile y México en niveles y en primeras diferencias. Se reportan las pruebas con tendencia e intercepto.

Las series bursátiles latinoamericanas en niveles generalmente presentan raíces unitarias, hecho confirmado por Brugger (2010) para las series en niveles del PIB y las respectivas bolsas analizadas en el presente estudio. Así, en los Cuadros 1 y 2 se reportan las pruebas de raíz unitaria sólo para las primeras diferencias. El Cuadro 1 indica que las series del crecimiento del piB de los países en la muestra son estacionarias. En todos los casos el rechazo a la hipótesis nula es fuerte. La estadística-t obtenida supera todos los valores críticos, señalando el rechazo de la existencia de raíz unitaria aun en el exigente nivel de confianza de 1\%. La probabilidad de un lado de MacKinnon confirma este resultado; en todos los casos es menor a $0.05 \%$. 
Samuel Brugger y Edgar Ortiz

Cuadro 1. Raíces unitarias de las series de PIB mensual de las economías latinoamericanas

\begin{tabular}{|c|c|c|c|c|c|}
\hline \multicolumn{6}{|c|}{ Primeras diferencias (crecimiento) } \\
\hline \multirow{7}{*}{$\begin{array}{l}\text { Augmented } \\
\text { Dickey-Fuller } \\
\text { test statistic }\end{array}$} & & Argentina & Brasil & Chile & México \\
\hline & & t-Statistic & t-Statistic & t-Statistic & t-Statistic \\
\hline & Valores críticos & -8.671863 & -3.517555 & -3.345139 & -3.371841 \\
\hline & $1 \%$ nivel & -3.476143 & -3.481217 & -3.476472 & -3.476472 \\
\hline & $5 \%$ nivel & -2.881541 & -2.883753 & -2.881685 & -2.881685 \\
\hline & $10 \%$ nivel & -2.577514 & -2.578694 & -2.577591 & -2.577591 \\
\hline & $\begin{array}{l}\text { Prob. (Mackinnon (1996) } \\
\text { one-sided p-values) }\end{array}$ & 0.000000 & 0.009000 & 0.014700 & 0.013600 \\
\hline
\end{tabular}

Fuente: Elaboración propia con datos obtenidos de CEPALStat y Economática.

El Cuadro 2 presenta para el crecimiento (primeras diferencias) de los índices bursátiles resultados similares a los obtenidos en las pruebas de raíces unitarias para el crecimiento de los PIB nacionales. Las series de los rendimientos de los mercados accionarios emergentes de Latinoamérica son estacionarias y no contienen raíz unitaria. En el caso de todos los mercados accionarios analizados la evidencia confirma la ausencia de raíces unitarias: el estadístico-t de la prueba de DAF es mayor que los valores críticos en todos los mercados para todos los niveles de confianza. El valor de la probabilidad de un lado de MacKinnon que tienen un valor igual a cero en todos los casos corrobora este hallazgo indicando para todos los niveles de confianza la ausencia de raíces unitarias en las series de rendimientos de los cuatro mercados de capital. Finalmente, cabe reconocer que para la prueba de las series en primera diferencia sólo se la aplicó con intercepto y se obtuvieron resultados favorables entre 6 a 10 rezagos. Cabe destacar que la mayoría de los estudios no sobrepasan en estos análisis los seis rezagos. Este resultado obtenido sobre la estacionaridad de las series es congruente con los resultados de los trabajos de Peiró (1996) y Ortiz et al. (2007). 
Mercados accionarios y su relación con la economía real en América Latina

Cuadro 2. Prueba de Raíces Unitarias de las series de índices y rendimientos bursátiles

\begin{tabular}{llcccc}
\hline \multicolumn{5}{c}{ Rendimientos (primeras diferencias) } \\
\hline & & Argentina & Brasil & Chile & México \\
\cline { 3 - 6 } & & t-Statistic & t-Statistic & t-Statistic & t-Statistic \\
\cline { 3 - 6 } & Valores críticos & -12.161390 & -12.184850 & -10.055380 & -11.064380 \\
$\begin{array}{l}\text { Augmented } \\
\text { Dickey-Fuller } \\
\text { test statistic }\end{array}$ & $1 \%$ nivel & -3.473096 & -3.473096 & -3.473096 & -3.473096 \\
& 5\% nivel & -2.880211 & -2.880211 & -2.880211 & -2.880211 \\
& $10 \%$ nivel & -2.576805 & -2.576805 & -2.576805 & -2.576805 \\
& $\begin{array}{l}\text { Prob. (Mackinnon (1996) } \\
\text { one-sided p-values) }\end{array}$ & 0.000000 & 0.000000 & 0.000000 & 0.000000 \\
\hline
\end{tabular}

Fuente: Elaboración propia con datos obtenidos de CEPalStat y Economática.

\section{Análisis de cointegración}

Aplicando el modelo de Johansen (ecs. 2, 3 y 4), obviando los resultados y análisis en niveles puesto que sus variables no son estacionarias, los resultados son favorables (Cuadro 3). Según la prueba de la traza se rechaza la hipótesis nula de la no existencia de vectores cointegrantes; el estadístico de la traza es mayor que los valores críticos a un nivel de $5.0 \%$ de significancia. Existen, en otras palabras, vectores de cointegración. Complementariamente, la prueba de la traza, cuyo estadístico es mayor que los valores críticos indica categóricamente que no hay más de un vector cointegrante. Considerando la prueba del máximo eingenvalue, los resultados son similares. El estadístico del máximo eingenvalue es mayor que el valor crítico a un nivel de 5\%; se rechaza la no existencia de vectores de cointegración; y se confirma, según el eingenvalue que no hay más de un vector de cointegración. En resumen, de acuerdo con la prueba de Johansen, los rendimientos de los índices bursátiles y los cambios porcentuales en el PIB sostienen una relación de equilibrio a largo plazo en los cuatro casos. 
Samuel Brugger y Edgar Ortiz

Cuadro 3: Análisis de cointegración, modelo de Johansen

\begin{tabular}{|c|c|c|c|c|}
\hline \multicolumn{5}{|c|}{ Argentina: Prueba de rango de cointegración (Traza) } \\
\hline Hipótesis & & Estadística & 0.05 & \\
\hline$N^{\circ} E C(s)$ & Eingenvalue & Traza & Valor crítico & Probabilidad $^{\star \star}$ \\
\hline Ninguno* & 0.415255 & 133.5876 & 15.49471 & 0.0001 \\
\hline No más de $1^{*}$ & 0.289855 & 52,02755 & 3.841466 & 0.0000 \\
\hline \multicolumn{5}{|c|}{ Argentina: Prueba de rango de cointegración sin restricción (Máximo eingenvalue) } \\
\hline Hipótesis & & & 0.05 & \\
\hline$N^{\circ} E C(s)$ & Eingenvalue & Máximo eigenvalue & Valor crítico & Probabilidad ${ }^{* \star}$ \\
\hline Ninguno* & 0.415255 & 81.56009 & 14.26460 & 0.0000 \\
\hline No más de $1^{*}$ & 0,289855 & 52.022755 & 3.841488 & 0.0000 \\
\hline \multicolumn{5}{|c|}{ Brasil: Prueba de rango de cointegración (Traza) } \\
\hline Hipótesis & & Estadística & 0.05 & \\
\hline$N^{\circ} E C(s)$ & Eingenvalue & Traza & Valor crítico & Probabilidad** \\
\hline Ninguno* & 0.274068 & 87.7402 & 15.49471 & 0.0000 \\
\hline No más de $1^{*}$ & 0.263961 & 42.90617 & 3.841466 & 0.0000 \\
\hline \multicolumn{5}{|c|}{ Brasil: Prueba de rango de cointegración sin restricción (Máximo eingenvalue) } \\
\hline Hipótesis & & & 0.05 & \\
\hline$N^{\circ} \mathrm{CE}(\mathrm{S})$ & Eingenvalue & Máximo eigenvalue & Valor crítico & Probabilidad $^{\star \star}$ \\
\hline Ninguno* & 0.274068 & 44.84184 & 14.26460 & 0.0000 \\
\hline No más de $1^{*}$ & .0263961 & 42.90617 & 3.841466 & 0.0000 \\
\hline \multicolumn{5}{|c|}{ Chile: Prueba de rango de cointegración sin restricción (Traza) } \\
\hline Hipótesis & & Estadística & 0.05 & \\
\hline$N^{\circ} E C(s)$ & Eingenvalue & Traza & Valor crítico & Probabilidad ${ }^{* *}$ \\
\hline Ninguno* & 0.434066 & 131.9731 & 15.49771 & 0.0001 \\
\hline No más de $1^{*}$ & 0.258390 & 45.43757 & 3.841466 & 0.0000 \\
\hline \multicolumn{5}{|c|}{ Chile: Prueba de rango de cointegración sin restricción (Máximo eingenvalue) } \\
\hline Hipótesis & & & 0.05 & \\
\hline$N^{\circ}(E(S)$ & Eingenvalue & Máximo eigenvalue & Valor crítico & Probabilidad ${ }^{\star *}$ \\
\hline Ninguno & 0.434066 & 86.53549 & 14.26450 & 0.0000 \\
\hline No más de 1 & 0.258390 & 45.43757 & 3.841466 & 0.0000 \\
\hline \multicolumn{5}{|c|}{ México: Prueba de rango de cointegración sin restricción (Traza) } \\
\hline Hipótesis & & Estadística & 0.05 & \\
\hline$N^{\circ} E C(s)$ & Eingenvalue & Traza & Valor crítico & Probabilidad $^{\star \star}$ \\
\hline Ninguno* & 0.541791 & 154.5115 & 15.49471 & 0.0001 \\
\hline No más de $1^{*}$ & 0.210292 & 35.88602 & 3.841466 & 0.0000 \\
\hline \multicolumn{5}{|c|}{ México: Prueba de rango de cointegración sin restricción (Máximo eingenvalue) } \\
\hline Hipótesis & & & 0.05 & \\
\hline$N^{\circ} E C(s)$ & Eingenvalue & Máximo eigenvalue & Valor crítico & Probabilidad ${ }^{* *}$ \\
\hline Ninguno* & 0.541791 & 118.6255 & 14.26460 & 0.0001 \\
\hline No más de $1^{*}$ & 0.210292 & 35.88602 & 3.841466 & 0.0000 \\
\hline \multicolumn{5}{|c|}{ *Indica rechazo de la hipótesis al nivel de 0.05.; ${ }^{* \star}$ MacKinnon-Haug-Michelis (1999) p-values } \\
\hline \multicolumn{5}{|c|}{ La Prueba de la traza indica 1 ecuación cointegrante (CE) al nivel de 0.05 . } \\
\hline \multicolumn{5}{|c|}{$\begin{array}{l}\text { La prueba del máximo eigenvalue indica } 2 \text { ecuaciones cointegrantes al nivel de } 0.05 \text {. } \\
\text { Fuente: Elaboración propia con base en resultados de Eviews } 6.0\end{array}$} \\
\hline
\end{tabular}


En la estimación de un análisis de largo plazo existen momentos de desequilibrio, desviaciones de corto plazo respecto a la relación a corto plazo justamente por el carácter aleatorio y complejo de las finanzas. Para identificar la magnitud y duración de dichos desequilibrios se utiliza el mecanismo de corrección de error (MCE) el cual permite vincular el análisis de equilibrio de largo plazo con la dinámica de ajuste de corto plazo, como una medida de desviación del equilibrio (ecs. 5 y 6 ).

Cuadro 4: MCE entre las tasas de crecimiento del PIB y del índice bursátil

\begin{tabular}{lcccc}
\hline & Argentina & Brasil & Chile & México \\
\hline Coeficiente mce & -0.02182 & -0.04712 & -0.00040 & -0.02796 \\
Std. Error & 0.00727 & 0.01233 & 0.01022 & 0.00669 \\
Prob. & 0.00320 & 0.00020 & 0.00080 & 0.00010 \\
R-squared & 0.94025 & 0.868723 & 0.960603 & 0.91399 \\
Durbin-Watson stat & 1.98152 & 1.951450 & 1.969353 & 2.089523 \\
\hline
\end{tabular}

Fuente: Elaboración propia con datos obtenidos de CEPALStat y Economática.

El Cuadro 4 muestra los resultados obtenidos, el término de error es bajo, indicando que las discrepancias entre el corto y el largo plazos son muy pequeñas. En el caso de Argentina, el coeficiente del error indica que alrededor de 2.1\% de la discrepancia negativa es reducida mes a mes; para Brasil hay un ajuste del 4.7\%; en Chile el ajuste es menor a $1 \%$ y en México es de alrededor de 3\%. Con la excepción de Chile, todos los países presentan un margen de error parecido. El coeficiente es significativo para los cuatro países al 1\%. Todos muestran una elevada $\mathrm{R}^{2}$; las estadísticas Durban-Watson de todas las regresiones son ligeramente mayores a 1.96, lo que indica ausencia de autocorrelación de los residuos.

\section{Análisis de causalidad Granger}

La prueba de causalidad de Granger (1969) busca determinar estadísticamente si el pasado de la variable $x$ contiene información que preceda al comportamiento de la variable $y$, y que, por tanto, contribuya a explicarla y viceversa. Cabe aclarar que la causalidad de Granger es muy sensible al número de rezagos. En el 
presente trabajo se encontró que para los cuatro países el número óptimo es de hasta 11 rezagos; 12 rezagos no son óptimos debido a la presencia del efecto diciembre en los cuatro mercados emergentes de AL (Brugger, 2009); esta anomalía genera grandes picos en los residuos y estadísticamente no es viable incluirlo en las autorregresiones. Los resultados se resumen en el Cuadro 5; se muestra el estadístico $F$ y su probabilidad en paréntesis. La hipótesis que se contrasta afirma que los coeficientes de las regresiones de $Y$ sobre $X$, así como los de $X$ sobre $Y$, son nulos para la variable de apoyo; es decir, que la variable $X$ no aporta información para explicar a $Y$, o bien, que $Y$ no aporta información para explicar a $X$. Si el valor del estadístico $F$ supera al valor tabulado, se rechaza la hipótesis nula y, por tanto, se aceptará que $X$ causa a $Y$ o viceversa.

Cuadro 5: Resultados de prueba de causalidad de Granger

\begin{tabular}{|c|c|c|c|c|c|}
\hline & & Argentina & Brasil & Chile & México \\
\hline \multirow[t]{2}{*}{$\begin{array}{l}\text { Causalidad de } \\
\text { la bolsa a la } \\
\text { economía real }\end{array}$} & 12 rezagos & $\begin{array}{c}1.78199 \\
(0.05877)\end{array}$ & $\begin{array}{l}\text { No hay } \\
\text { causalidad }\end{array}$ & $\begin{array}{l}\text { No hay } \\
\text { causalidad }\end{array}$ & $\begin{array}{l}2.65066 \\
(0.00353)\end{array}$ \\
\hline & 11 rezagos & $\begin{array}{l}4.99838 \\
(2.2 \mathrm{E}-06)\end{array}$ & $\begin{array}{c}2.43215 \\
(0.00948)\end{array}$ & $\begin{array}{c}2.51102 \\
(0.00700)\end{array}$ & $\begin{array}{c}3.33439 \\
(0.00049)\end{array}$ \\
\hline $\begin{array}{l}\text { Causalidad de la } \\
\text { economía real } \\
\text { hacia la bolsa }\end{array}$ & 11 rezagos & $\begin{array}{c}1.13170 \\
(0.34279)\end{array}$ & $\begin{array}{c}0.88616 \\
(0.55616)\end{array}$ & $\begin{array}{c}0.63631 \\
(0.79469)\end{array}$ & $\begin{array}{c}1.14822 \\
(0.33057)\end{array}$ \\
\hline $\begin{array}{l}\text { Análisis con } \\
\text { series en moneda } \\
\text { original }\end{array}$ & $\begin{array}{l}\text { Tanto con } 12 \text { como } \\
\text { con } 11 \text { rezagos }\end{array}$ & No hay diferencia & No hay diferencia & No hay diferencia & № hay diferencia \\
\hline
\end{tabular}

Fuente: Elaboración propia con datos obtenidos de CEPalStat y Economática.

La causalidad Granger identificada en el Cuadro 5 es unidireccional: del índice bursátil al PIB mensual; esto es, no se encontró causalidad en dirección opuesta. Esto significa que la bolsa influye en el desarrollo económico pero éste no influye en el desempeño de la bolsa. Este resultado sugiere que la actividad bursátil en los mercados accionarios emergentes de AL sigue circuitos propios desligados de la economía real: no se basa en información pasada de los fundamentos de la economía y se basa más bien en tendencias bursátiles pasadas, en expectativas de la propia bolsa así como en la especulación. Una explicación plausible sobre esta unilateralidad debe encontrarse en la importancia de la información bursátil rezagada en los mercados emergentes a falta de una amplia información sobre los fundamentos de la economía. 
Finalmente, en el Cuadro 5 también se reportan los resultados de la prueba de causalidad Granger utilizando las series en moneda local, prueba realizada para determinar si el tipo de cambio afecta los resultados. No se encontraron resultados diferentes. La unidireccionalidad de la relación bolsa-mercado confirma que la evidencia presentada es afín a los resultados obtenidos por estudios de Christopoulos y Tsionas (2003), Hassapis y Kalyvitis (2002), Howells y Soliman $(2003 ; 2005)$ y Nasseh y Strauss (2000). Por su parte, este resultado rechaza los obtenidos por Apergis, Filippidis y Economidou (2007), Tuncer y Alovsat (2000) y et al. (2007c), en los que existe una bidireccionalidad, así como el de Fama (1990), quien argumenta que la relación es exactamente en sentido contrario.

\section{Vectores autorregresivos}

Los análisis de VAR (ecs. 8, 9, 10) han ganado mucha popularidad en los últimos años, principalmente debido a las restricciones y críticas que han recibido los modelos tradicionales. La metodología var de Sims (1980), (ecs 9, 10 y 11) está estrechamente ligada con la cointegración que se analizó en la sección anterior por el teorema de representación de Granger, el cual indica que si existe cointegración debe haber necesariamente una representación de esta relación de largo plazo en una de corto plazo, que corrige el error que le es consustancial y evita que las series cointegradas se dispersen en el tiempo. Pero el teorema también plantea que la cointegración es una condición necesaria para que se pueda realizar un modelo de corrección de error. De esta forma, se combina la idea de encontrar una relación estable de largo plazo con los ajustes estadísticos de los desequilibrios de corto plazo. De hecho, el análisis de cointegración resuelve con solvencia el problema o desequilibrio original de los VAR, el hecho de contar con poco fundamento teórico.

Para estimar el VAR es preciso identificar el grado de integración de las variables; sólo si presenta el mismo orden de integración se contará con información histórica relevante que no se debe perder. Como en el caso actual las series en niveles son I(1) y no cointegran, se optará por analizar sólo las series en primeras diferencias. Otro factor importante del análisis var es el número de rezagos a emplearse, por lo que es necesario evaluar uno a uno cada rezago hasta encontrar el número óptimo. Con tal propósito es indispensable contrastar los criterios de información estadística de los resultados que genera cada especificación. La mayoría de los paquetes econométricos calculan un gran número de criterios estadísticos: prueba LR, error de predicción final (FPE), Hannan-Quinn, 
Akaike y Schwarz. En la presente tesis se utilizarán estos dos últimos criterios que son los más utilizados en la literatura financiera. En general muestran un resultado muy parecido o incluso idéntico, pero ocasionalmente pueden dar resultados muy distintos. En este caso se tomará el criterio que ofrece menos rezagos. Esto debido a que -como lo demuestra Loría (2007) - un incremento de rezagos disminuye $\mathrm{R}^{2}$.

Otra característica importante es la estabilidad dinámica del var, la que se puede observar mediante el valor de sus raíces características. Con esto se asegura que ante choques de corto plazo las variables regresen a su trayectoria de equilibrio de largo plazo. Si no se diera el caso, se tendría un modelo explosivo, lo que no tendría sentido económico. Para verificar, entonces, la estabilidad se deben analizar las raíces características $\lambda n$, las cuales deben ser menores a 1 en su valor absoluto: $ц \lambda n ц<1$. En el Cuadro 6 se puede observar que el óptimo para los cuatro var es 11 rezagos, lo cual no debe sorprender en vista de lo mencionado en la sección anterior.

Cuadro 6. Definición de rezagos para el análisis VAR

\begin{tabular}{lccccccc}
\hline & Rezagos & LogL & $L R$ & FPE & AIC & SC & HQ \\
\hline Argentina & 11 & -947.1980 & $29.43972^{\star}$ & $3375.099^{\star}$ & $13.79442^{\star}$ & 14.74311 & $14.17991^{\star}$ \\
Brasil & 11 & -1086.419 & $60.53101^{\star}$ & $97664.17^{\star}$ & $17.15786^{\star}$ & 18.16247 & $17.56609^{\star}$ \\
Chile & 11 & -852.7923 & $127.7309^{\star}$ & $909.5857^{\star}$ & $12.48323^{\star}$ & $13.43192^{\star}$ & $12.86872^{\star}$ \\
México & 11 & -855.1101 & $34.13096^{\star}$ & $939.3442^{\star}$ & $12.51542^{\star}$ & 13.46411 & $12.90091^{\star}$ \\
\hline
\end{tabular}

*Indica el orden de rezago seleccionado por el criterio.

LE: criterio estadístico LR secuencial modificado (toda prueba al nivel de 5\%); FPE: Error de predicción final; AlC: Criterio de Aikake; SC: Criterio de información de Schwarz; HQ: Criterio de información de Nannan-Quinn.

Fuente: Elaboración propia con datos obtenidos de CEPalStat y Economática.

Una vez definido el modelo var se debe analizar si pasa pruebas tan básicas como normalidad, heteroscedasticidad y estabilidad. En el Cuadro 7 se muestran los resultados obtenidos para los países más representativos de AL.

Tanto la normalidad como la heteroscedasticidad deben pasar las mismas condiciones como si fueran estimaciones mediante MCO, es decir, su probabilidad debe ser mayor a 0.05 , lo que equivale a que el valor del estadístico $F$ supere el valor tabulado; entonces se puede rechazar la hipótesis nula de no normalidad o no heterescedasticidad. La estabilidad, por su lado, analiza las raíces características, las cuales deben ser menores a 1 , o dicho de forma geométrica, deben estar dentro del círculo. 
Cuadro 7. Pruebas de especificación correcta del VAR

\begin{tabular}{|c|c|c|c|c|c|}
\hline & & Argentina & Brasil & Chile & México \\
\hline Normalidad & Jarque-Bera conjunta & $\begin{array}{r}8.006857 \\
(0.0913)\end{array}$ & $\begin{array}{r}6.504773 \\
(0.1645)\end{array}$ & $\begin{array}{c}12.44381 \\
(0.0143)^{\star}\end{array}$ & $\begin{array}{r}16.67677 \\
(0.0540)^{\star \star}\end{array}$ \\
\hline Heteroscedasticidad & Chi-sq conjunto & $\begin{array}{r}156.4989 \\
(0.0716)\end{array}$ & $\begin{array}{r}148.2914 \\
(0.1575)\end{array}$ & $\begin{array}{r}138.5762 \\
(0.3303)\end{array}$ & $\begin{array}{r}160.1598 \\
(0.0581)\end{array}$ \\
\hline Estabilidad & $\begin{array}{l}\text { Raíces del polinomial } \\
\text { característico }\end{array}$ & $\begin{array}{l}\text { Ninguna raíz } \\
\text { yace fuera del } \\
\text { círculo de unidad } \\
\text { var satisface } \\
\text { la condición de } \\
\text { estabilidad }\end{array}$ & $\begin{array}{l}\text { Ninguna raíz } \\
\text { yace fuera del } \\
\text { círculo de unidad } \\
\text { var satisface } \\
\text { la condición de } \\
\text { estabilidad. }\end{array}$ & $\begin{array}{l}\text { Ninguna raíz } \\
\text { yace fuera del } \\
\text { círculo de unidad } \\
\text { var satisface } \\
\text { la condición de } \\
\text { estabilidad }\end{array}$ & $\begin{array}{l}\text { Ninguna raíz } \\
\text { yace fuera del } \\
\text { círculo de unidad } \\
\text { var satisface } \\
\text { la condición de } \\
\text { estabilidad }\end{array}$ \\
\hline
\end{tabular}

* Muestra problemas de curtosis: 12.1847 (0.00230).

** Muestra problemas en la asimetría estadística: 7.641913 (0.0219).

Fuente: Elaboración propia con datos obtenidos de CEPalStat y Economática.

Gráfica 3. Círculo de raíces características

\section{Panel (a) Argentina}

Inverse Roots of AR Characteristic Polynominal

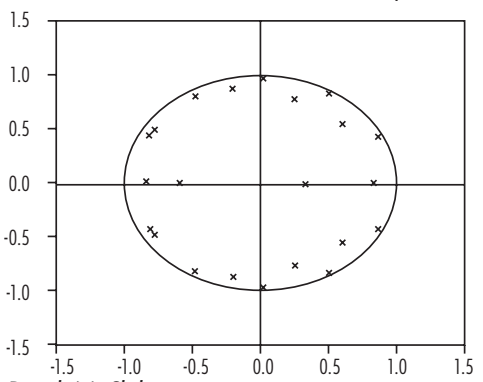

Panel (c) Chile

Inverse Roots of AR Characteristic Polynominal

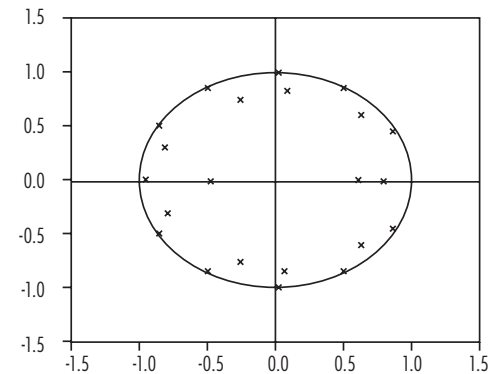

Panel (b) Brasil

Inverse Roots of AR Characteristic Polynominal

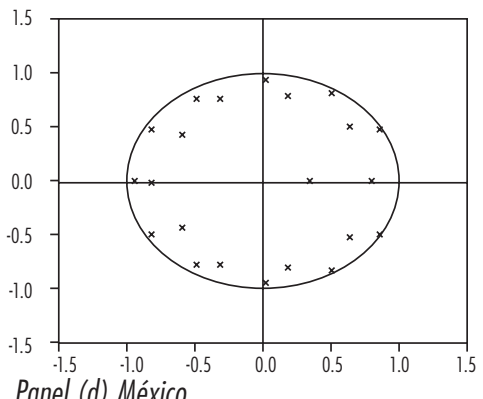

Panel (d) México

Inverse Roots of AR Characteristic Polynominal

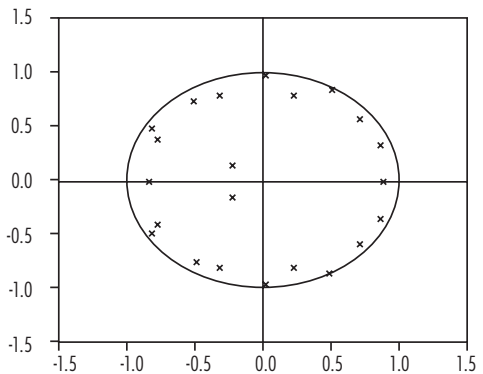

Fuente: Elaboración propia con datos obtenidos de CEPALStat y Economática. 
Samuel Brugger y Edgar Ortiz

\section{Análisis impulso-respuesta}

El análisis impulso-respuesta es un instrumento útil para evaluar la congruencia y la sensibilidad dinámica de las variables especificadas en un modelo, cuidando que no hayan covarianzas distintas a 0 , es decir, errores correlacionados, ya que en ese caso sería imposible precisar la respuesta de las variables ante impulsos de variables específicas (Pindyck y Rubinfeld, 2001). El análisis indica la respuesta dinámica de la variable dependiente en el var ante choques en los términos de error o innovaciones de todas las variables endógenas, excluyendo los efectos de las variables que expresamente se asignan como exógenas (ecs 11 y 12). Cabe resaltar que la respuesta al impulso solamente puede ser calculada si el var se encuentra en equilibrio de largo plazo. Por otro lado, se debe considerar la longitud del choque, ya que si éste se considera muy corto no se puede observar con precisión la evolución de los choques ni la estabilidad dinámica del var. De esta manera, si el var es estable, una perturbación hará que el sistema salga de su trayectoria de equilibrio, aunque después de algunos periodos vuelva al equilibrio.

Los análisis del presente estudio se basan en un intervalo de tres años a fin de observar el proceso de choque y ajuste de manera completa. Aunque el análisis impulso-respuesta genera cuatro análisis -todas las variables contra todas-, únicamente se examina el impulso generado por las bolsas de valores, ya que, como se mencionó en la sección de causalidad de Granger, es sólo esta relación la estadísticamente significativa y que es precisamente la pregunta que se quiere contestar, esto es, si las bolsas latinoamericanas impactan el desarrollo económico. En la Gráfica 4 se observan los cuatro casos.

En el panel (a), en el caso de Argentina los 36 meses son estadísticamente significativos al 5\% y prácticamente todos al 1\%. El impulso del MERval comienza positivo aunque decreciente durante los primeros tres meses; el impacto en el PIB se vuelve negativo en el cuarto mes y positivo nuevamente en el quinto mes y después zigzaguea para llegar a la estabilidad. Destacan únicamente el impacto positivo del octavo mes y el considerable impacto negativo del décimosegundo mes.

En el panel (b) se muestra el análisis impulso-respuesta del BOvespa para la economía brasileńa. Al igual que en el caso argentino, prácticamente todos los periodos son significativos al $1 \%$ y todos son significativos al 5\%. En los primeros dos meses existe una respuesta positiva de casi la misma magnitud, que se vuelve negativa en los meses tercero y cuarto. Posteriormente se estabiliza en 0 hasta el noveno mes, cuando el mercado accionario tiene un impacto positivo de casi la misma magnitud que durante los primeros dos meses, para después volverse negativo en el doceavo mes. Más tarde toma una forma zigzagueante para llegar a su punto estable. 
Gráfica 4. Análisis impulso-respuesta de los rendimientos de las bolsas y las economías latinoamericanas
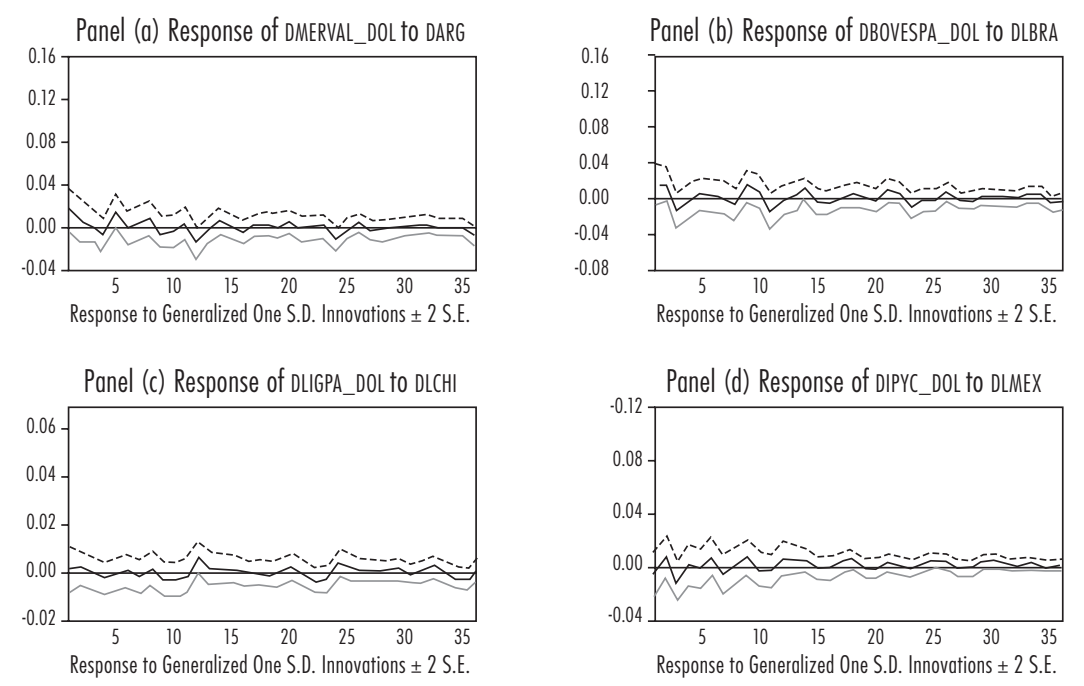

Fuente: Elaboración propia con datos obtenidos de CEPALStat y Economática.

El panel (c) resume el impulso-respuesta del IgPa al piв mensual de Chile. En este caso, todos los periodos son significativos al 1\%. El impacto es ligeramente positivo durante los dos primeros meses y luego ligeramente negativos hasta el mes décimosegundo en que se observa un impacto positivo. Posteriormente los impactos son zigzagueantes pero muy pequeños. Consiguientemente, es posible afirmar que la bolsa chilena sólo tiene un impacto mínimo en la economía real, lo que en cierta medida contradice la hipótesis de que el mercado bursátil es esencial para el desarrollo. Considerado particularmente, el mercado bursátil chileno es el más desarrollado en Latinoamérica; así, el alto y ejemplar desarrollo de la economía chilena ha estado prácticamente desligado de la actividad bursátil del país.

Por último, en el panel (d) se representa el caso mexicano. Todos los periodos son significativos al $1 \%$. La respuesta, aunque sea muy débil -al igual que en Chile-, es interesante ya que es contraria a la de los demás países. Comienza en el primer periodo con un impacto negativo, que se vuelve positivo en el segundo mes y negativo en el tercero. Posteriormente comienza a desaparecer el impulso y zigzaguea hasta llegar a la estabilidad. 
Samuel Brugger y Edgar Ortiz

\section{Análisis de descomposición de la varianza}

La descomposición de la varianza es un estudio complementario al análisis impulso-respuesta que informa en distintos horizontes del tiempo el porcentaje de volatilidad que registra una variable por los choques de las demás. De esta manera es posible medir la volatilidad que genera la variable endógena a la variable exógena en un momento específico. En el Cuadro 8 se resumen los resultados para el primer y trigésimo sexto periodo. Se observa que todas las variables tienen un fuerte comportamiento autorregresivo, en virtud de que después de 36 meses más del $90 \%$ de la varianza de cada variable se sigue explicando por sí misma, excepto en el caso de México. Además, ninguna de las variables pierde capacidad explicativa rápidamente con sus propios rezagos. Como es irrelevante la relación de la economía real con la economía financiera, dichos resultados no se analizan. Sin embargo, a la inversa sí es relevante. En Argentina, el MERVAL explica el $1.2 \%$ en el primer periodo y se incrementa a $7 \%$ en el periodo 36 . En Brasil en el primer periodo el BOvEspa sólo se explica el 0.9\% y en el periodo 36 se explica el 7.27\% de la varianza del PIB mensual. En Chile el IGPA explica en el primer periodo el $0.01 \%$ y en el periodo 36 el 3.32\% de la varianza. Por último, en México el IPYC explica el $0.5 \%$ de la varianza en el primer periodo y $5.8 \%$ en el 36 .

Cuadro 8. Descomposición de la varianza de la tasa de crecimiento del PIB y de los rendimientos bursátiles

\begin{tabular}{lcccccccc}
\hline & ARG & MERVAL & BRA & BOVESPA & CHI & IGPA & MEX & IPYC \\
\hline 1 periodo & 100 & 98.08517 & 100 & 98.81114 & 100 & 99.97552 & 100 & 99.41169 \\
36 periodos & 91.24916 & 91.91923 & 95.41204 & 92.22507 & 95.63607 & 96.43409 & 75.20432 & 94.36168
\end{tabular}

Fuente: Elaboración propia con datos obtenidos de CEPalStat y Economática.

Otra manera de contrarrestar la correcta especificación del modelo consiste en revisar la magnitud del error estándar de cada ecuación para los periodos considerados, esto es, el grado de dispersión de las estimaciones puntuales obtenidas en muestras de un tamańo determinado. Un mismo estimador ofrece distintos valores para diversas muestras del mismo tamaño extraídas de la misma población. Por lo tanto, se debe tener una medida de la variabilidad del estimador respecto del parámetro que se trata de estimar. En el Cuadro 9 se muestran los resultados. Se observa en primer lugar que el error estándar es mayor en la variable bursátil que en el piB mensual, lo cual concuerda con la teoría. Sin embargo, 
sobresale la diferencia del error estándar entre el MERVAL, el BOVESPA y el IPYC en relación al IGPA de Chile, donde se experimentan pocas turbulencias, tiene un error estándar mucho más bajo (6\%) que el de las demás bolsas (Argentina, 12\%; Brasil, 13\%, y México, 10\%). En el IPYC es donde más aumenta el error estándar, mientras que en el caso de las demás bolsas se mantiene estable.

Cuadro 9. Error estándar de la tasa de crecimiento del PIB y de los rendimientos bursátiles

\begin{tabular}{lcccccccc}
\hline & ARG & MERVAL & BRA & Bovespa & CHI & IGPA & MEX & IPyC \\
\hline 1 periodo & 0.03569 & 0.12550 & 0.03823 & 0.13730 & 0.02555 & 0.06078 & 0.01646 & 0.10779 \\
36 periodo & 0.05921 & 0.13612 & 0.06353 & 0.14492 & 0.04913 & 0.06625 & 0.030253 & 0.14792
\end{tabular}

Fuente: Elaboración propia con datos obtenidos de CEPalStat y Economática.

\section{CONCLUSIONES}

Aunque los rendimientos accionarios de los principales mercados de AL mantienen una relación de equilibrio con el PIB, en gran medida esta relación es débil. Las series de tiempo del pIB y de la actividad bursátil en sus primeras diferencias no cumplen estrictamente los criterios de normalidad para los casos de Argentina, Brasil, Chile y México. Sin embargo, los diferentes análisis econométricos evidencian que los rendimientos de los mercados accionarios de estos países y el crecimiento de su producción agregada sí mantienen una relación de equilibrio a largo plazo. Durante el periodo analizado, 1993 a 2005, las desviación mensual de dicho equilibrio fue relativamente pequeña en todos los casos, negativa para Argentina, $-2.1 \%, 4.7 \%$ en el caso de Brasil, menor a $1 \%$ en el caso de Chile, y alrededor de 3\% en el caso de México. El análisis de causalidad Granger confirma una relación unidireccional de la Bolsa al pIB en todos los casos, no encontrándose sorprendentemente una relación bidireccional como es común en muchas economías estudiadas. El análisis de vectores autorregresivos confirma los hallazgos sobre cointegración y corrección del error a corto plazo, en tanto que las funciones de impulso respuesta indican para todos los casos, excepto para México un ligero impulso positivo de la bolsa al pIB; en el caso de México el impulso es ligeramente negativo; en todos los casos la reacción zigzaguea y se estabiliza en alrededor de 15 meses. Finalmente la descomposición de la varianza demuestra que los errores estándar bursátiles son mayores que el error estándar del PIB y que las desviaciones del equilibrio pueden presentarse desde el primer mes hasta el último del periodo analizado. 
El hecho de que la dirección de causalidad entre la actividad bursátil y la economía real en Latinoamérica (al menos en los países estudiados) sea sólo unidireccional, del comportamiento bursátil a la economía real, es preocupante pues señala una total autonomía de la actividad bursátil en relación a la economía real. Aunque inicialmente se pueda identificar un impacto positivo debido a los altos niveles de inversión extranjera, esto implica que al tomar en cuenta las tendencias del mercado accionario las decisiones sobre inversión, ahorro, consumo y producción por parte de los grupos familiares, el gobierno y las empresas productivas siguen pasos en falso puesto que la actividad bursátil está desligada de la economía real. El hecho de que la actividad bursátil siga circuitos propios, sin tomar en cuenta el desempeño y necesidades de la economía real, señala que el financiamiento accionario al crecimiento empresarial es exiguo; la actividad bursátil se limita al mercado secundario y propenso a la especulación. En suma, el Consenso de Washington y la globalización económico-financiera han propiciado la liberalización de las cuentas de capital, induciendo un alto crecimiento de los mercados financieros, en particular de los mercados accionarios, pero sin medidas complementarias que vinculen dicho crecimiento con la economía real. Se tiene un impacto aparentemente positivo hacia la economía real (a través de las reservas internacionales). No obstante sólo queda como el efecto de un indicador adelantado con un efecto positivo en el pIB, pero que pronto se diluye por la falta de vinculación de la actividad bursátil con la economía empresarial. Tres importantes lecciones que ameritan futuras investigaciones y sobre las cuales se deben desarrollar nuevas políticas de desarrollo bursátil son: la vinculación, efectos contagio y dependencia de los mercados emergentes de AL con los mercados accionarios de los países desarrollados; la formulación de políticas de regulación que eviten la especulación y la presencia de "inversiones golondrina" en los mercados de capital emergentes; y la vinculación entre la actividad bursátil y la inversión real de las empresas. 


\section{BIBLIOGRAFÍA}

Athanasios, V.y A. Adamopoulos, "Financial Development and Economic Growth", en American Journal of Applied Science, vol. 6, num. 11, 2009, pp. 1410-1417.

Atje, R. and B. Jovanovic, "Stock Markets and Development", en European Economic Review, vol. 37, num. 2-3, 1993, pp. 632-640.

Binswanger, M., "Stock Returns and Real Activity. Is There Still a Connection?", en Applied Financial Economics, vol. 10, num. 4, 2000, pp. 379-87.

Binswanger, M., "How do Stock Prices Respond to Fundamental Shocks?, en Research Letters, vol. 1, num. 2, 2004a, pp. 90-99.

Binswanger, M., "Stock Markets and Real Activity in the G-7 Countries: Did the Relationship Change During the 1980's?", en The Quarterly Journal of Economics and Finance 44(2), 2004b, pp. 237-252.

Brugger, S., "El contagio financiero. Una modelación econométrica mediante SARIMAX”, Documento de investigación, Facultad de Economía, unam, 2009.

Brugger, S. y E. Ortiz, "Mercados de capital y crecimiento económico en Latinoamérica”, Documento de investigación, Facultad de Economía, UNAM, 2010.

Capasso, S., "Stock Market Development and Economic Growth: Tales of Informational Asymmetries", en Journal of Economic Surveys, vol. 18, num. 2, 2004, pp. 267-292.

Caporale, G. M., P. G. A. Howells, y A. M. Soliman, "Stock Market Development and Economic Growth: The Causal Linkage", en Journal of Economic Development, vol. 29, num. 1, 2003, pp. 33-50.

Charemza, W. W. y M. Blangiewicz, "East European Economic Reform: Some Simulations on a Structural var Model", en Journal of Policy Modelling, vol. 23, 2001, pp. 147-160.

Choong, C. K., Z. Yusop, S. H. Law y V. L. K. Sen, "Financial Development and Economic Growth in Malaysia: The Stock Market Perspective", en Investment Management and Financial Innovations, 4, 2005, pp.105-115.

Demirgüc-Kunt, A. and Levine, R., "Stock Market, Corporate Finance and Economic Growth: An Overview", en The World Bank Economic Review, vol. 10, num. 2, 1996, pp. 223-239.

Dickey, D. y W. Fuller, "Distribution of the Estimators for Autoregressive Time Series with Unit Root", Journal of the American Statistical Association, vol. 74(336), 1979, pp. 427-431.

Engle, R. F. and C. W. J. Granger, "Co-integration and Error Correction: Representation, Estimation and Testing", Econometrica, vol. 55, num. 2, 1987, 251-276. 
Samuel Brugger y Edgar Ortiz

Enisan, A. A. y A. O. Olufisayo, "Stock Market Development and Economic Growth; Evidence from Seven Sub-Saharan African Countries", en Journal of Economics and Business, vol. 61, 2009, pp. 162-171.

Fama, E., "Stock Returns and Real Activity", en The Journal of Finance, vol. 45, num. 4, 1990, 1089-1108.

Goldsmith, R. W., Financial Structure and Development, Yale University Press, 1960.

Granger, C. W. J., "Investigating Causal Relations by Econometric Models and Cross-Spectral Methods", en Econométrica, vol. 37, num 3, 1969, pp. 424-438.

Gurley, J. G. and Shaw, E. S., "Financial Aspects of Economic Development", en American Economic Review, vol. 45, num. 4, 1955, pp. 515-538.

Gurley, J. G. and E. S. Shaw, Money in a Theory of Finance, Washington, Brookings Institution, 1960.

Hassapis, C. and S. Kalyvitis, "Investigating Links Between Growth and Real Stock Price Changes with Empirical Evidence from the G-7 Countries", The Quarterly Review of Economics and Finance, vol. 42, num. 3, 2002, pp. 543-575.

Johansen, S., "Estimation and Hypothesis Testing of Cointegration in Gaussian Vector Autoregressive Models", Econométrica, vol. 59, num. 6, 1991, pp. 1551-1580.

Johansen, S., Likehood-Based Inference in Cointegrated Autoregressive Models, Oxford, Oxford University Press, 1995.

Levine, R., "Financial Development and Economic Growth: Views and Agenda”, en Journal of Economic Literature, vol. 35, 1997, pp. 688-726.

Levine, R. and S. Zervos, "Capital Control Liberalization and Stock Market Development”, World Development, vol. 26, num. 7, 1998, pp. 1169-1183.

Mauro, P., "Stock Returns and Output Growth in Emerging and Advanced Economies", en Journal of Development Studies, vol. 71, num. 1, 2003, pp. 129-153.

MacKinnon, J. G., "Numerical Distribution Functions for Unit Root and Cointegration Tests", en Journal of Applie Econometrics, vol. 11, num. 6, 1996, pp. 601-618.

MacKinnon, J. G., A. Haug y L. Michelis, "Numerical Distribution Functions of Likehood Ratio Tests for Cointegration", en Journal of Applied Econometrics, vol. 14, num. 5, 1999, pp. 563-577.

MacKinnon, R. I., Money and Capital in Economic Development, Brookings Institution, 1973.

Mishkin, F. S., The Economics of Money, Banking and Financial Markets, Addison Wesley Longman, 1973. 
Nasseh, A. y J. Strauss, "Stock Prices and Domestic and International Activity: A Cointegration Approach", The Quarterly Reviews of Economics and Finance, vol. 40, num. 2, 2000, pp. 229-245.

Ortiz, E., A. Cabello y R. de Jesús, “The Role of Mexico's Stock Exchange in Economic Growth", en The Journal of Economic Asymmetries, vol. 4, num. 2, 2007, pp. 1-26.

Peiró, A., "Stock Prices, Production and Interest Rates; Comparison of Three European Countries with the USA", en Empirical Economics, vol. 21, 1996, pp. 221-234.

Pindyck, R. S. y D. L. Rubinfeld, Econometría. Modelos y pronósticos, McGraw Hill, México, 2001.

Sargan, J. D., "Wages and Prices in the United Kingdom: A Study in Econometric Methodology", in Hart, P. E., , G. Mills and J. K. Whitaker (eds.), Econometric Analysis for National Economic Planning, Vol. 16 of Colston Papers, London, Butterworlds, 1964, pp. 25-54; reimpreso en Walls, K. F. y D. F. Hendry (eds.), Econometrics and Quantitative Economics Analysis, New York, Basil Blackwell, 2000.

Shaw, E. S., Financial Deepening in Economic Development, Oxford University Press, 1973.

Sims, C. A., "Macroeconomics and Reality", en Econometrica, vol. 48, num. 1, pp.1-48.

Singh, A., "Financial Liberalization, Stock Markets and Economic Development”, Economic Journal, vol. 107, num. 3, 1997, pp. 771-782.

Singh, A. y B. Weiss, "Emerging Stock Markets, Portfolio Capital Flows and Long-Term Economic Growth: Micro and Macro Perspectives", en World Development, vol. 29, 1998, pp. 607-622.

Stiglitz, J. E., "Credit Markets and the Control of Capital", Journal of Money, Credit and Banking, vol. 17. num. 2, 1985, pp. 133-152.

Stiglitz, J. and Weiss, A., "Credit Rationing in Markets with Imperfect Information", American Economic Review, vol. 7, num. 2, 1981, pp. 393-410.

Tuncer, G. C. y M. Alövsat, "Stock Markets and Economic Growth: A Causality Test”, en Dugus University Journal 2 (Turquía), 2000, pp. 124-132. 